\title{
Variational approach to KPZ: Fluctuation theorems and large deviation function for entropy production
}

Cite as: Chaos 30, 073107 (2020); https://doi.org/10.1063/5.0006121

Submitted: 17 March 2020. Accepted: 11 June 2020. Published Online: 02 July 2020

Horacio S. Wio (D), Miguel A. Rodríguez (D), and Rafael Gallego (D)

\section{COLLECTIONS}

Paper published as part of the special topic on Instabilities and Nonequilibrium Structures

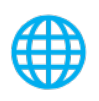

\section{ARTICLES YOU MAY BE INTERESTED IN}

Competing local and global interactions in social dynamics: How important is the friendship network?

Chaos: An Interdisciplinary Journal of Nonlinear Science 30, 073105 (2020); https:// doi.org/10.1063/5.0004797

A note on the continuity for Caputo fractional stochastic differential equations Chaos: An Interdisciplinary Journal of Nonlinear Science 30, 073106 (2020); https: // doi.org/10.1063/1.5141485

Chaotic motion due to lateral Casimir forces during nonlinear actuation dynamics Chaos: An Interdisciplinary Journal of Nonlinear Science 30, 073101 (2020); https:// doi.org/10.1063/5.0011334

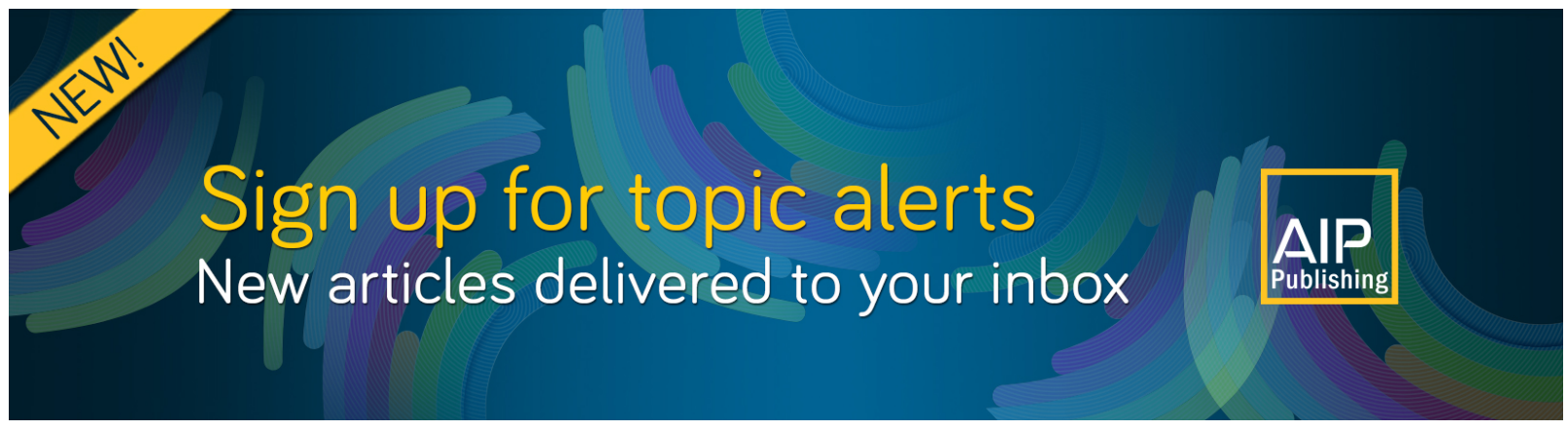




\title{
Variational approach to KPZ: Fluctuation theorems and large deviation function for entropy production
}

Cite as: Chaos 30, 073107 (2020); doi: $10.1063 / 5.0006121$
Submitted: 17 March $2020 \cdot$ Accepted: 11 June 2020.
Published Online: 2 July 2020

Horacio S. Wio, , ,a) (iD Miguel A. Rodríguez, ${ }^{2}$ (D) and Rafael Gallego ${ }^{3}$ (i)

\author{
AFFILIATIONS \\ ${ }^{1}$ IFISC (Instituto de Física Interdisciplinar y Sistemas Complejos), Universitat de les IIles Balears-CSIC, \\ 07122 Palma de Mallorca, Spain \\ ${ }^{2}$ Instituto de Física de Cantabria (IFCA), CSIC-UNICAN, E-39005 Santander, Spain \\ ${ }^{3}$ Mathematics Department, Gijón Campus, Universidad de Oviedo, E-33203 Gijón, Spain
}

Note: This article is part of the Focus Issue, instabilities and Nonequilibrium Structures.

a) Author to whom correspondence should be addressed: horacio@ifisc.uib-csic.es

\begin{abstract}
Motivated by the time behavior of the functional arising in the variational approach to the Kardar-Parisi-Zhang (KPZ) equation, and in order to study fluctuation theorems in such a system, we have adapted a path-integral scheme that adequately fits to this kind of study dealing with unstable systems. As the KPZ system has no stationary probability distribution, we show how to proceed for obtaining detailed as well as integral fluctuation theorems. This path-integral methodology, together with the variational approach, in addition to allowing analyze fluctuation theorems, can be exploited to determine a large deviation function for entropy production.
\end{abstract}

Published under license by AIP Publishing. https://doi.org/10.1063/5.0006121

\begin{abstract}
Nonequilibrium systems have attracted a growing research interest during the last decades. Among the many aspects, there are two that have been extensively studied. On the one hand, there is the paradigmatic Kardar-Parisi-Zhang (KPZ) model that describes the growth of rough surfaces for out-of equilibrium extended systems. On the other hand, there is stochastic thermodynamics that intends to describe, exploiting stochastic variables, the non-equilibrium dynamics present in microscopic as well as mesoscopic systems. Here, both aspects have been integrated in order to analyze the first from the point of view of the second. It is shown how the variational approach of the KPZ system allows one to readily obtain fluctuation theorems as well as information about the large deviation function for the entropy production. It is expected that such an approach could offer alternative information about critical exponents for the KPZ universality class.
\end{abstract}

\section{INTRODUCTION}

The last few decades have witnessed a growing interest in diverse aspects of nonequilibrium systems. Among them, there are two that we want to address in this paper. The first aspect is related to a paradigmatic model: the Kardar-Parisi-Zhang (KPZ) equation, ${ }^{1-4}$ a simple (albeit nontrivial) representation describing the growth of rough surfaces of an out-of equilibrium extended system. For the one-dimensional (1D) case, and during the last couple of decades, researchers have found several amazing exact results (for instance, to indicate just a few, see Refs. 5 and 6). Some recent review-like articles could give an idea of the state of the art regarding the $\mathrm{KPZ}$ equation and its critical properties. ${ }^{7,8}$ Additionally, a novel variational approach was introduced in Refs. 9-11.

The second aspect refers to the so-called stochastic thermodynamics, ${ }^{12-17,19,20}$ exploiting continuous (via Langevin-like equations) as well as discrete (via master equations) descriptions and approaches, where some recent review-like articles give a panoramic view of the field and the broad spectrum of fluctuation theorems that were obtained as well as of their possible applications. ${ }^{21,22}$

Until very recently, these two aspects have not been analyzed together, that is, studies on the stochastic thermodynamics for the KPZ model are scarce. ${ }^{23-25}$ At variance with the one dimensional study just done in Ref. 24, we want here to exploit the variational approach indicated above and introduced in Refs. 9 and 11. 
An extremely interesting aspect of such an approach is that it gives valid results in all dimensions.

In order to fix ideas, let us start remembering the form of the KPZ equation,

$$
\begin{aligned}
\frac{\partial}{\partial t} h(\bar{r}, t) & =v \nabla^{2} h(\bar{r}, t)+\frac{\lambda}{2}(\nabla h(\bar{r}, t))^{2}+F+\xi(\bar{r}, t) \\
& =-\frac{\delta}{\delta h} \Phi[h]+\xi(\bar{r}, t) .
\end{aligned}
$$

The functional indicated on the second line was obtained in Refs. 9 and 11 and has the form

$$
\begin{aligned}
\Phi[h]= & \frac{v}{2} \int d \bar{r}(\nabla h(\bar{r}, t))^{2}-\int d \bar{r} F h(\bar{r}, t) \\
& -\frac{\lambda}{2} \int d \bar{r} \int_{0}^{t} d s \partial_{s} h(\bar{r}, s)(\nabla h(\bar{r}, s))^{2} .
\end{aligned}
$$

This functional is the one we will work with. In what follows, and in order to simplify this first approach, we will adopt $F \equiv 0$ for the external field.

On the one hand, in 11 , it was shown that $\Phi[h]$ has a linear dependence with time, while on the other hand, the above indicated exact (1D) studies ${ }^{5,6}$ indicate the existence of neither a stationary nor a steady state solution for the probability distribution function (PDF). It is worth noting that such a PDF keeps memory of the initial condition (a fact that arises naturally within the variational approach ${ }^{11}$ ). Aspects of such a peculiar behavior were analyzed in Ref. 29 through the study of some toy models. Those ideas are the ones we will elaborate in the present work.

In Sec. II, and exploiting a path-integral scheme, ${ }^{14,18,19}$ we will define expressions for the probability of entropy production (EP) along a trajectory, and derive both, detailed and integral fluctuation theorems. The most relevant aspect is that those relations results to be connected with $\Phi[h]$, the nonequilibrium potential (NEP) arising within the variational approach indicated before., ${ }^{9,11}$ After that, we make connection with the Large Deviation Function (LDF) ${ }^{30}$ for entropy production. Again, the result is expressed in terms of $\Phi[h]$. Section $V$ is devoted to discuss the results, extract some conclusions, and propose some future lines of work.

\section{FLUCTUATION THEOREMS}

In order to build up a stochastic thermodynamics for the KPZ system by exploiting the variational formalism, we will apply the path-integral scheme, ${ }^{18}$ somehow following Ref. 19. As a first step, we define the probability of following a given (fixed) trajectory from $\left(h_{a}\left(\vec{r}, t_{a}\right), t_{a}\right)$ up to $\left(h_{b}\left(\vec{r}, t_{b}\right), t_{b}\right)$ [in what follows, $\left(h_{a}, t_{a}\right)$ up to $\left.\left(h_{b}, t_{b}\right)\right]$ by

$$
P^{F}\left[h_{b}, t_{b} \mid h_{a}, t_{a}\right]=\mathfrak{N}^{F} e^{-S^{F}[h(t)]} .
$$

Here, $\mathfrak{N}^{F}$ is a normalization factor, and the forward stochastic action $S^{F}[h]$ is

$$
S^{F}[h]=\int_{t_{a}}^{t_{b}} d s \mathfrak{L}^{F}(h, \dot{h}),
$$

with $\mathfrak{L}^{F}(h, \dot{h})$ being the forward stochastic Lagrangian given by

$$
\mathfrak{L}^{F}(h, \dot{h})=\frac{1}{4 \varepsilon} \int d \bar{r}\left[\dot{h}-\left[v \nabla^{2} h(\bar{r}, t)+\frac{\lambda}{2}(\nabla h(\bar{r}, t))^{2}\right]\right]^{2} .
$$

As indicated in Ref. 25, we do not explicitly state an expression for the Jacobian ensuing from the variable transformation from noises to heights, as it turns out that such a Jacobian does not contribute to the entropy relations.

For the inverse or backward trajectories, we have

$$
P^{B}\left[h_{b}, t_{b} \mid h_{a}, t_{a}\right]=\mathfrak{N}^{B} e^{-S^{B}[h(t)]},
$$

with $\mathfrak{N}^{B}$ being a normalization factor (in general, $\mathfrak{N}^{F}=\mathfrak{N}^{B}=\mathfrak{N}$ ); the backward stochastic action

$$
S^{B}[h]=\int_{t_{a}}^{t_{b}} d s \mathfrak{L}^{B}(h, \dot{h})
$$

and its backward stochastic Lagrangian

$$
\mathfrak{L}^{B}(h, \dot{h})=\frac{1}{4 \varepsilon} \int d \bar{r}\left[\dot{h}+\left[v \nabla^{2} h(\bar{r})+\frac{\lambda}{2}(\nabla h(\bar{r}, t))^{2}\right]\right]^{2} .
$$

As is usual in stochastic thermodynamics, and following Ref. 16, the increase of entropy of the medium (interchange entropy) associated with a trajectory from $\left(h_{a}, t_{a}\right)$ up to $\left(h_{b}, t_{b}\right)$ is defined as

$$
R^{F}[h]=\ln \frac{P^{F}\left[h_{b}, t_{b} \mid h_{a}, t_{a}\right]}{P^{B}\left[h_{b}, t_{b} \mid h_{a}, t_{a}\right]},
$$

where $P^{F, B}\left[h_{b}, t_{b} \mid h_{a}, t_{a}\right]$, as indicated before, is the probability of the indicated trajectory. Hence, as the interchange entropy $R^{F}$ along the referred trajectory is given by the logarithm of the ratio between the forward and backward probabilities, we will need the difference between the stochastic actions $\triangle S=S^{F}[h]-S^{B}[h]$ that, from the previous expressions, results as

$$
S^{F}[h]-S^{B}[h]=-\frac{1}{\varepsilon} \int d \bar{r} \int_{t_{a}}^{t_{b}} d t\left(\partial_{t} h\right)\left[\nu \nabla^{2} h(\bar{r})+\frac{\lambda}{2}(\nabla h(\bar{r}, t))^{2}\right],
$$

extending a result in Ref. 20. On the one hand, the first term on the rhs can be written as

$$
\begin{aligned}
- & \frac{v}{\varepsilon} \int d \bar{r} \int_{t_{a}}^{t_{b}} d t\left(\partial_{t} h\right) \nabla^{2} h(\bar{r})=\frac{v}{\varepsilon} \int d \bar{r} \int_{t_{a}}^{t_{b}} d t\left(\nabla \partial_{t} h\right)(\nabla h) \\
=\frac{v}{2 \varepsilon} \int d \bar{r} \int_{t_{a}}^{t_{b}} d t \partial_{t}(\nabla h)^{2} & =\frac{v}{2 \varepsilon} \int d \bar{r}\left[\left(\nabla h_{b}\right)^{2}-\left(\nabla h_{a}\right)^{2}\right]
\end{aligned}
$$

while on the other hand, the time integral in the second term, going from $t_{a}$ up to $t_{b}$, could be separated into two parts: an integral from $t_{0}$ up to $t_{b}$ minus another that goes from $t_{0}$ up to $t_{a}$, as follows:

$$
\begin{aligned}
\int d \bar{r} \int_{t_{a}}^{t_{b}} d t \partial_{t} h(\nabla h(\bar{r}, t))^{2}= & \int d \bar{r} \int_{t_{o}}^{t_{b}} d t \partial_{t} h(\nabla h(\bar{r}, t))^{2} \\
& -\int d \bar{r} \int_{t_{o}}^{t_{a}} d t \partial_{t} h(\nabla h(\bar{r}, t))^{2} .
\end{aligned}
$$


Putting everything together, we obtain

$$
\begin{aligned}
& S^{F}[h]-S^{B}[h]=\frac{v}{2 \varepsilon} \int d \bar{r}\left[\left(\nabla h_{b}\right)^{2}-\left(\nabla h_{a}\right)^{2}\right] \\
& \quad-\frac{\lambda}{2 \varepsilon} \int d \bar{r} \int_{t_{a}}^{t_{b}} d t \partial_{t} h(\nabla h(\bar{r}, t))^{2}=\frac{1}{\varepsilon}\left(\Phi\left[h_{b}\right]-\Phi\left[h_{a}\right]\right) .
\end{aligned}
$$

When $\lambda=0$ [i.e., Edwards-Wilkinson (EW) case], it agrees with the result in Ref. 20, but for $\lambda \neq 0$, it clearly differs, as includes a KPZ contribution which is not only valid for any dimension but is also not restricted to periodic boundary conditions.

Hence, we have the following relation [see Eq. (32) in Ref. 19]:

$$
\frac{P^{F}\left[h_{b}, t_{b} \mid h_{a}, t_{a}\right]}{P^{B}\left[h_{a}, t_{a} \mid h_{b}, t_{b}\right]}=e^{-\left(\Phi\left[h_{b}\right]-\Phi\left[h_{a}\right]\right) / \varepsilon}=e^{R^{F}\left[h_{a}, h_{b}\right]},
$$

where

$$
R^{F}\left[h_{a}, h_{b}\right]=-\frac{1}{\varepsilon}\left(\Phi\left[h_{b}\right]-\Phi\left[h_{a}\right]\right),
$$

with $R^{F}$ being the above defined interchange entropy, as in Refs. 16 and 24. The result in Eq. (12) offers further support to our argument that the functional $\Phi[h]$ is the "NEP" for KPZ.

For reasons that will be apparent in a while, and calling $P_{1}\left(h_{a}, t_{a}\right)$ and $P_{2}\left(h_{b}, t_{b}\right)$ to the PDFs at times $t_{a}$ and $t_{b}$, respectively, we introduce the quantity

$$
R_{0}=-\ln \left(\frac{P_{1}\left(h_{a}, t_{a}\right)}{P_{2}\left(h_{b}, t_{b}\right)}\right),
$$

corresponding to the entropy increment associated with the formation of the states $h_{a}$ and $h_{b}$. Note that the definition of state entropy in stochastic thermodynamics is $s(h, t)=-\ln P(h, t)$. We also define the joint probabilities $\hat{P}^{F}\left(h_{b}, t_{b} \mid h_{a}, t_{a}\right)=P_{1}\left(h_{a}, t_{a}\right)$ $P^{F}\left(h_{b}, t_{b} \mid h_{a}, t_{a}\right)$ and $\hat{P}^{B}\left(h_{a}, t_{a} \mid h_{b}, t_{b}\right)=P_{2}\left(h_{b}, t_{b}\right) P^{B}\left(h_{a}, t_{a} \mid h_{b}, t_{b}\right)$, with $P^{F}$ and $P^{B}$ being the forward and backward propagator, respectively, that in terms of path integrals are given by

$$
P^{F, B}\left(h_{b}, t_{b} \mid h_{a}, t_{a}\right)=\int_{h_{a}}^{h_{b}} \mathfrak{D}[h] P^{F, B}\left[h_{b}, t_{b} \mid h_{a}, t_{a}\right] .
$$

For the following steps, it will be necessary to explicitly introduce the form of $P_{1}\left(h_{a}, t_{a}\right)$ and $P_{2}\left(h_{b}, t_{b}\right)$. Clearly, the direct way to get it is to consider the (non-steady state) probability distribution function (PDF) given by

$$
P_{1}\left(h_{a}, t_{a}\right)=\int_{\Omega} d \bar{r} P\left(h_{a}, t_{a} \mid \hat{h}, t=0\right) P_{o}(\hat{h}(\bar{r})),
$$

with $\Omega$ indicating the $d$-dimensional space $(d=1,2, \ldots)$ where we are working and $P_{o}(\hat{h}(\bar{r}))$ is the (completely arbitrary, usually assumed to be plane) initial condition at $t_{0}=0 . P\left(h_{a}, t_{a} \mid \hat{h}, t=0\right)$ is the propagator. It could be convenient to choose $t_{a}>t^{*}$, where $t^{*}$ is the time at which we reach the maximum of $\Phi[h]$ as function of $t$, as shown in Figs. 1 and 5 of Ref. 11. As for $t<t^{*}$, we will essentially be within the Edwards-Wilkinson (EW) regime (clearly, the crossover EW-KPZ has been extensively studied and a few examples are Refs. 26-28). Anyway, times $t_{a, b}$ can be chosen in a complete arbitrary way.

From Eq. (14), it follows that

$$
\begin{aligned}
P_{2}\left(h_{b}, t_{b}\right)= & \int_{\Omega} d \bar{r} P\left(h_{b}, t_{b} \mid h_{a}(\bar{r}), t_{a}\right) P_{1}\left(h_{a}(\bar{r}), t_{a}\right) \\
= & \int_{\Omega} d \bar{r} \int_{\Omega} d \overline{r^{\prime}} P\left(h_{b}, t_{b} \mid h_{a}(\bar{r}), t_{a}\right) \\
& \tilde{P}\left(h_{a}(\bar{r}), t_{a} \mid \hat{h}\left(\overline{r^{\prime}}\right), t=0\right) P_{o}\left(\hat{h}\left(\overline{r^{\prime}}\right)\right)
\end{aligned}
$$

and $\left(h_{b}, t_{b}\right),\left(h_{a}, t_{a}\right)$ are fixed. However, due to the enormous difficulties of obtaining even a simple approximate histogram for $P_{1}\left(h_{a}, t_{a}\right)$ or $P_{2}\left(h_{b}, t_{b}\right)$, it results more adequate to make and "educated guess" about their form and work with—the so far-arbitrary (approximate) choices for those PDFs.

In order to obtain other useful theorems and/or relations, as in Eq. (40) of Ref. 19, we will consider a beam of trajectories, with $R$, a fixed value of the total EP, that originates at the initial condition $\left(h_{a}, t_{a}\right)$ chosen from a fixed $P_{1}\left(h_{a}, t_{a}\right)$ and trajectories ending at $\left(h_{b}, t_{b}\right)$; while for the backward process, we consider as initial condition $\left(h_{b}, t_{b}\right)$ chosen from a fixed $P_{2}\left(h_{b}, t_{b}\right)$, with trajectories that ends at $\left(h_{a}, t_{a}\right)$. However, $P_{2}\left(h_{b}, t_{b}\right)$ does not necessarily result from the evolution of $P_{1}\left(h_{a}, t_{a}\right)$ and vice versa, $P_{1}\left(h_{a}, t_{a}\right)$ does not result from the backward evolution starting at $P_{2}\left(h_{b}, t_{b}\right)$, i.e., they are completely arbitrary.

We use the previous results (as well as the properties of the Dirac's $\delta$ ) to get the trajectory's beam as

$$
\begin{aligned}
\varrho^{F}\left(R, h_{a}, h_{b}\right) & =P_{1}\left(h_{a}, t_{a}\right) \int_{h_{a}}^{h_{b}} \mathfrak{D}[h] P^{F}\left[h_{b}, t_{b} \mid h_{a}, t_{a}\right] \delta\left(R^{F}\left[h_{a}, h_{b}\right]+R_{0}-R\right) \\
& =P_{1}\left(h_{a}, t_{a}\right) \int_{h_{a}}^{h_{b}} \mathfrak{D}[h] e^{R^{F}\left[h_{a}, h_{b}\right]} P^{B}\left[h_{a}, t_{a} \mid h_{b}, t_{b}\right] \delta\left(R^{F}\left[h_{a}, h_{b}\right]-\left(R-R_{0}\right)\right) \\
& =P_{1}\left(h_{a}, t_{a}\right) \int_{h_{a}}^{h_{b}} \mathfrak{D}[h] e^{R-R_{0}} P^{B}\left[h_{a}, t_{a} \mid h_{b}, t_{b}\right] \delta\left(R^{F}\left[h_{a}, h_{b}\right]-\left(R-R_{0}\right)\right) \\
& =P_{2}\left(h_{b}, t_{b}\right) e^{R} \int_{h_{a}}^{h_{b}} \mathfrak{D}[h] P^{B}\left[h_{a}, t_{a} \mid h_{b}, t_{b}\right] \delta\left(R^{F}\left[h_{a}, h_{b}\right]-\left(R-R_{0}\right)\right)
\end{aligned}
$$




$$
\begin{aligned}
& =e^{R} P_{2}\left(h_{b}, t_{b}\right) \int_{h_{a}}^{h_{b}} \mathfrak{D}[h] P^{B}\left[h_{a}, t_{a} \mid h_{b}, t_{b}\right] \delta\left(R^{B}\left[h_{b}, h_{a}\right]+\left(R-R_{0}\right)\right) \\
& =e^{R} \varrho^{B}\left(-R, h_{a}, h_{b}\right)
\end{aligned}
$$

where $R=R^{F}+R_{0}$ is the total entropy or entropy production (EP), and the normalization is implicit and included in the measure $\mathfrak{D}[h]$. Hence, we have

$$
\frac{\varrho^{F}\left(R, h_{a}, h_{b}\right)}{\varrho^{B}\left(-R, h_{a}, h_{b}\right)}=e^{R},
$$

where for $R$, we have a fixed unique value and, as indicated before, $P_{1}\left(h_{a}, t_{a}\right)$ and $P_{2}\left(h_{b}, t_{b}\right)$ are also fixed PDFs. It is worth remembering that $R^{F}=-\frac{1}{\varepsilon}\left(\Phi\left[h_{b}\right]-\Phi\left[h_{a}\right]\right)$ is evaluated along the forward trajectory and, due to the odd symmetry under time reversal, also fulfills a relation with $R^{B}$ (the backward EP): $R^{B}\left[h_{b}, h_{a}\right]=-R^{F}\left[h_{a}, h_{b}\right.$ ] [as indicated in Eq. (39) in Ref. 19].

Now, operating similarly as in the case before, we can also define the probability $P(R)$ of having a value $R$ for the total EP as (remember $R$ is fixed)

$$
\begin{aligned}
P(R)= & \int d h_{a} \int d h_{b} P_{1}\left[h_{a}, t_{a}\right] \int_{h_{a}}^{h_{b}} \mathfrak{D}[h] P^{F}\left[h_{b}, t_{b} \mid h_{a}, t_{a}\right] \delta\left(R^{F}\right. \\
& \left.+R_{0}-R\right)=\int d h_{a} \int d h_{b} \varrho^{F}\left(R, h_{a}, h_{b}\right)
\end{aligned}
$$

resulting in the detailed fluctuation relation ${ }^{21}$

$$
P(R)=e^{R} P(-R),
$$

which is tightly related to the Crook theorem. ${ }^{15}$ It is worth remarking here that $P_{1}$ and $P_{2}$ are arbitrary.

Exploiting Eq. (18), we define the mean value

$$
\begin{aligned}
\left\langle e^{-\hat{R}\left[h_{a}, h_{b}\right]}\right\rangle= & \int_{h_{a}} d h_{a} P_{1}\left[h_{a}, t_{a}\right] \int_{h_{b}} d h_{b} \\
& \times \int \mathfrak{D}[h] P^{F}\left[h_{b}, t_{b} \mid h_{a}, t_{a}\right] e^{-R^{F}\left[h_{a}, h_{b}\right]+\ln \left[P_{2}\left(h_{b}, t_{b}\right) / P_{1}\left(x_{a}, t_{a}\right)\right]},
\end{aligned}
$$

with $\quad \hat{R}^{F}\left[h_{a}, h_{b}\right]=R^{F}\left[h_{a}, h_{b}\right]+R_{0} \quad$ (and obviously, $\quad \hat{R}^{B}\left[h_{a}, h_{b}\right]$ $\left.=R^{B}\left[h_{a}, h_{b}\right]-R_{0}\right)$. Using Eq. (12), the last expression transforms into

$$
\begin{aligned}
\left\langle e^{-\hat{R}\left[h_{a}, h_{b}\right]}\right\rangle & =\int_{h_{a}} d h_{a} P_{1}\left[h_{a}, t_{a}\right] \int_{h_{b}} d h_{b} \int \mathfrak{D}[h] P^{F}\left[h_{b}, t_{b} \mid h_{a}, t_{a}\right] e^{-\hat{R}^{F}\left[h_{a}, h_{b}\right]} \\
& =\int_{h_{a}} d h_{a} \int_{h_{b}} d h_{b} P_{2}\left(h_{b}, t_{b}\right) \int \mathfrak{D}[h] P^{B}\left[h_{b}, t_{b} \mid h_{a}, t_{a}\right] \equiv 1 .
\end{aligned}
$$

Hence, we have

$$
\left\langle e^{-\hat{R}\left[h_{a}, h_{b}\right]}\right\rangle \equiv 1,
$$

which agrees with the standard form of the integral theorem.

\section{LARGE DEVIATION FUNCTION}

In this section, we will look for information on the Large Deviation Function $(\mathrm{LDF})^{30}$ for the PDF of the entropy production. There are several recent papers related to the LDF for KPZ (to indicate just a few, see Refs. 31-35). However, in general, most of them (if not all) consider such a LDF for the maximum height at a given point and only in the one-dimensional case.

Here, we will exploit a simple approach in order to obtain the indicated information on the LDF for the PDF of the EP. We start with an idea that was used in Ref. 11 and giving further support to the scaling found in Ref. 24, indicating that the LDF, in any dimension, behaves as $\zeta(R) \sim \lambda^{2}$.

As is known, the definition of the LDF is ${ }^{30}$

$$
\zeta(R)=-\lim _{t \rightarrow \infty} \frac{1}{t} \ln [P] .
$$

We start remembering Eq. (19) and also taking into account that the position of the mean value of $P_{1}\left(h, t_{a}\right)$ and $P_{2}\left(h, t_{b}\right)$ should typically evolve as $\left\langle h\left(t_{a, b}\right)\right\rangle \sim v_{\infty} t_{a, b}$ (and $v_{\infty} \sim \lambda$ ), those PDFs will have widths that depend on the chosen forms.

It is useful to rewrite Eq. (19) as

$$
\ln [P(R)]=\ln [P(-R)]+R .
$$

Now, as was observed in Ref. 24, $P(R)$ slightly departs from a Gaussian (in Ref. 24, it was analyzed by considering a Gaussian plus an higher order approximation), particularly showing some degree of skewness. It is a solidly grounded argument based on the result indicated above as well as on the behavior of $\left\langle\Phi\left[h_{b}\right]\right\rangle$ vs $t$, as shown in Ref. 11, that such a quasi-Gaussian character, slightly corrected for skewness, could be extended to higher dimensions (and valid for any value of $\lambda$ ). Hence, we can write

$$
P(R) \approx \exp \left\{-\left(R-R_{m} t\right)^{2} / 4 R_{m} t\right\}
$$

with a fast decaying skewness correction.

From Eq. (23), we readily have

$$
\begin{aligned}
\zeta(R) & =-\lim _{t_{b} \rightarrow \infty} \frac{1}{t_{b}}(R+\ln [P(-R)]) \\
& =-\lim _{t_{b} \rightarrow \infty} \frac{1}{t_{b}} \ln [P(-R)] \\
& =-\lim _{t_{b} \rightarrow \infty} \frac{-1}{t_{b}} \frac{\left(R+R_{m} t_{b}\right)^{2}}{4 R_{m} t_{b}} \\
& =\frac{R_{m}}{4}=\frac{\langle R\rangle}{4 t_{b}}
\end{aligned}
$$


and the indicated average results

$$
\begin{aligned}
\langle R\rangle & \simeq\left\langle R^{F}\left[h_{a}, h_{b}\right]+R_{0}\right\rangle \\
& \simeq \frac{-1}{\varepsilon}\left(\left\langle\Phi\left[h_{b}\right]\right\rangle-\left\langle\Phi\left[h_{a}\right]\right\rangle\right)-\lambda\left(\Lambda_{b} t_{b}-\Lambda_{a} t_{a}\right),
\end{aligned}
$$

with $\Lambda_{a, b}$ being some constant that will depend on the choice adopted for $P_{i}(i=1,2)$.

Hence, results from Ref. 11 [see Sec. 4, Eqs. (16) and (17)], the analysis of the rhs of Eq. (23) leads, up to the dominant order in $t$, to

$$
\frac{1}{\varepsilon}\left(\left\langle\Phi\left[h_{b}\right]\right\rangle-\left\langle\Phi\left[h_{a}\right]\right\rangle\right) \sim-\frac{2}{\varepsilon} \lambda^{2}\left(\Theta_{b} t_{b}-\Theta_{a} t_{a}\right),
$$

where $\Theta_{a, b}$ indicate the remaining contributions besides the $\lambda^{2}$ dependence. Clearly, we have here used the observed linear dependence of $\langle\Phi[h]\rangle$ with time. In a "general" sense, we can guess that

$$
\Theta_{a, b} \approx\left\langle\int_{h_{a, b}} d \bar{r}\left(\nabla h_{a, b}\right)^{2}\right\rangle=\left\langle\overline{\left(\nabla h_{a, b}\right)^{2}}\right\rangle
$$

with \langle\rangle implying an ensemble average and the overline indicating space averaging.

If we use the above indicated definition of $\zeta(R)$, we find that on the lhs of Eq. (26), the second term is much smaller than the first one ${ }^{15}$ while on the rhs of Eq. (25), the fourth term goes as $t_{a} / t_{b}$ that, for $t_{b} \gg t_{a}$, goes to zero. Finally, we have

$$
\zeta(R)=-\lim _{t_{b} \rightarrow \infty} \frac{\langle R\rangle}{t_{b}} \sim \lambda^{2} \Theta_{b}+\lambda \Lambda_{b}
$$

and, as indicated before, the result is valid for any value of $\lambda$; hence, the influence of the second term will become apparent only for $\lambda \ll 1$.

\section{LIMITATIONS TO THE USUAL SCALING}

It is tempting to exploit a very well known assumption, valid for one dimensional systems, ${ }^{3}$ to higher dimensions. For the moment, we left out the $R_{0}$ contribution. Such an assumption corresponds to the scaling

$$
h(x, t) \approx v_{\infty} t+(\Gamma t)^{\beta} \chi\left(x^{\prime}\right),
$$

where $x^{\prime}=A x / 2(\Gamma t)^{1 / z}, A=v / 2 D, \Gamma=A^{2} \lambda / 2$, and in $d=1$, the exponents are $\alpha=1 / 2, \beta=1 / 3$, and $z=3 / 2$. In addition, $v_{\infty}=D \lambda / 4 v l$, with $l$ being the lattice cut-off. We can make the "reasonable" (even natural) proposal that, for higher dimensions, we could generalize the indicated asymptotic form as

$$
h(\bar{r}, t) \approx v_{\infty} t+(B t)^{a} \chi\left(\bar{r}^{\prime}\right),
$$

with $\bar{r}^{\prime}=A \bar{r} / 2(B t)^{b}$ and the coefficients $A$ and $B$ as well as the exponents $a$ and $b$ to be determined. However, it also results obviously reasonable to assume $a=\beta$ and $b=1 / z$.

Keeping in mind that we still have the contribution coming from $R_{0}$ (that we are not considering here), as indicated in Eq. (25), we are interested in the behavior of $\Phi[h]$, as indicated in Eq. (2) or, alternatively, in

$$
\partial_{t} \Phi[h]=-\int d \bar{r}\left(v \nabla^{2} h+\frac{\lambda}{2}(\nabla h)^{2}\right) \partial_{t} h,
$$

whose study is clearer, simpler, and more direct. In order to extract the time dependence, we need to transform the diverse contributions from the old $(\bar{r})$ to the new $\left(\bar{r}^{\prime}\right)$ variables. The details are shown in the Appendix. The relevant time dependence that we find is

$$
\begin{aligned}
& \text { (1) }=\Psi_{1} t^{a-b d}+\Psi_{2} t^{2 a-b d}, \\
& \text { (2) }=\Psi_{3} t^{2 a-1-b d}+\Psi_{4} t^{3 a-1-b d}, \\
& \text { (3) }=\Psi_{5} t^{2 a-1-b-b d}+\Psi_{6} t^{-1-b-b d},
\end{aligned}
$$

and it is worth indicating that $\Psi_{2} \sim \frac{v^{2} \lambda^{2}}{D^{2} l}$.

In order to have a term that is both independent of time and having a $\lambda^{2}$ dependence, in Eq. (29), we should have that

$$
2 a-b d \equiv 0, \quad \Rightarrow \beta=d / 2 z,
$$

but such a relation leads immediately to $\alpha=d / 2$ that for $d=2$ leads to $\alpha=1$. Hence, the extension of the above indicated scaling to higher dimensions, even looking reasonable, leads to wrong results. Besides, in Ref. 36, exploiting this kind of scaling, a two dimensional case was analyzed. However, the system's symmetry is such that it is reduced to an effective one-dimensional situation.

\section{CONCLUSIONS}

We have shown that, based on a path-integral approach, it is possible to prove several fluctuation theorems for the KPZ system, a paradigmatic nonequilibrium extended model. The analysis exploits the knowledge and properties of $\Phi[h]$, the NEP for KPZ, as studied in Ref. 11. It is worth remarking that when proving both the detailed theorem Eq. (19) and the integral theorem Eq. (22) and due to the tremendous difficulties (for not saying the impossibility) for obtaining even an approximate histogram for $P_{1}\left(h_{a} ; t_{a}\right)$ and $P_{2}\left(h_{b} ; t_{b}\right)$, we have to consider arbitrary forms for those initial and final PDFs.

In addition to the fluctuation theorems, and using a kind of phenomenological approach, we have shown the form of the LDF for EP that results in also being related to $\Phi[h]$ and indicates that it should, essentially, scale as $\lambda^{2}$ in all dimensions.

The numerical analysis of the detailed theorem Eq. (19) (by considering arbitrary, however reasonable, forms of $P_{1}$ and $P_{2}$ ) as well as the LDF scaling, together with the analysis of possible relations allowing us to determine-or at least to give a range to bound-the critical exponents (for instance, as numerically evaluated in Refs. 37-39) or to determine general thermodynamic uncertainty relations ${ }^{25}$ will be the subject of forthcoming work.

\section{AUTHORS' CONTRIBUTIONS}

All the authors contribute equally to this work.

\section{ACKNOWLEDGMENTS}

H.S.W. thanks the IFISC for the kind hospitality extended to him, and fruitful discussions with R. R. Deza, J. A. Revelli, and R. Garcia-Garcia. M.A.R. and R.G. acknowledge support from the 
Ministerio de Economia y Competitividad (MINECO), Spain, under Project No. Fis2016-74957-P. We dedicate this work to the memory of Professor Enrique Tirapegui, a great physicist and best friend.

\section{APPENDIX: CHANGE OF VARIABLES}

As indicated above, in order to extract the time dependence, we need to transform the diverse contributions from the old $(\bar{r})$ to the new $\left(\bar{r}^{\prime}\right)$ variables. The Jacobian associated with such a transformation $\bar{r} \rightarrow \bar{r}^{\prime}$, in dimension $d$, is

$$
\mathbb{J}=\left(\partial \vec{r}^{\prime} / \partial \bar{r}\right)=\left(\frac{A}{2(B t)^{b}}\right)^{d} .
$$

The different contributions to be transformed are (i) $\partial_{t} h(\bar{r}, t)$, (ii) $\nabla^{2} h(\bar{r}, t)$, and (iii) $(\nabla h(\bar{r}, t))^{2}$. Hence, we have

$$
\begin{gathered}
\text { (i) } \partial_{t} h(\bar{r}, t)=v_{\infty}+a B(B t)^{a-1} \chi\left(\vec{r}^{\prime}\right) \\
-(B t)^{a} \mathbb{J} \frac{A B b}{2(B t)^{b+1}}\left[\vec{r}^{\prime} \cdot \nabla^{\prime} \chi\left(\vec{r}^{\prime}\right)\right], \\
\text { (ii) }(\nabla h(\bar{r}, t))^{2}=(B t)^{2 a} \mathbb{J}^{2}\left(\nabla \chi\left(\vec{r}^{\prime}\right)\right)^{2}, \\
\text { (iii) } \nabla^{2} h(\bar{r}, t)=(B t)^{a} \mathbb{J}^{2} \nabla^{2} \chi\left(\bar{r}^{\prime}\right)=(B t)^{a} \mathbb{J}^{2} \triangle^{\prime} \chi\left(\bar{r}^{\prime}\right),
\end{gathered}
$$

where $\Delta=\nabla^{2}$, and the symbol ' indicates that the differential operator acts on the variable $\bar{r}^{\prime}$. Now, replacing this expressions into the one for $\partial_{t} \Phi[h]$, we get the following contributions:

$$
\begin{aligned}
\text { (1) }- & \int d \vec{r}^{\prime} v_{\infty} \frac{A^{d}}{2(B t)^{d b}}(B t)^{a}\left(v \Delta^{\prime} \chi+\frac{\lambda}{2}(B t)^{a}(\nabla \chi)^{2}\right) \\
& =-v_{\infty} \frac{A^{d}}{2 B^{d b}} B^{a} t^{a-b d} \int d \vec{r}^{\prime}\left(v \triangle^{\prime} \chi+\frac{\lambda}{2}(B t)^{a}(\nabla \chi)^{2}\right) \\
& =\Psi_{1} t^{a-b d}+\Psi_{2} t^{2 a-b d}, \\
\text { (2) }- & \int d \vec{r}^{\prime} a B \frac{A^{d}}{2(B t)^{b d}}(B t)^{a-1} \chi\left(v \Delta^{\prime} \chi+\frac{\lambda}{2}(B t)^{a}(\nabla \chi)^{2}\right) \\
& =-a B^{2 a} \frac{A^{d}}{2 B^{b d}} t^{2 a-1-b d} \int d \vec{r}^{\prime}\left(v \Delta^{\prime} \chi+\frac{\lambda}{2}(B t)^{a}(\nabla \chi)^{2}\right) \\
& =\Psi_{3} t^{2 a-1-b d}+\Psi_{4} t^{3 a-1-b d}, \\
(3) \quad & d \vec{r}^{\prime}(B t)^{2 a} \frac{A^{d}}{2(B t)^{d b}} \frac{A B b}{2}(B t)^{-b-1}\left(\vec{r}^{\prime} \cdot \nabla^{\prime} \chi\right) \\
\times & \left(v \triangle^{\prime} \chi+\frac{\lambda}{2}(B t)^{a}(\nabla \chi)^{2}\right)=B^{2 a-b-b d}\left(\frac{A}{2}\right)^{d} \frac{A b}{2} t^{2 a-1-b d-b} \\
\times & \int d \vec{r}^{\prime}\left(v \triangle^{\prime} \chi+\frac{\lambda}{2}(B t)^{a}(\nabla \chi)^{2}\right)=\Psi_{5} t^{2 a-1-b-b d} \\
+ & \Psi_{6} t^{3 a-1-b-b d},
\end{aligned}
$$

where we have explicitly indicated the time dependence, and the dependence on the rest of parameters is absorbed into $\Psi_{i}$.

\section{DATA AVAILABILITY}

Data sharing is not applicable to this article as no new data were created or analyzed in this study.

\section{REFERENCES}

${ }^{1}$ M. Kardar, G. Parisi, and Y. C. Zhang, "Dynamic scaling of growing interfaces," Phys. Rev. Lett. 56, 889 (1986)

${ }^{2}$ T. Halpin-Healy and Y.-C. Zhang, "Kinetic roughening phenomena, stochastic growth, directed polymers and all that," Phys. Rep. 254, 215 (1995).

${ }^{3}$ J. Krug, "Origins of scale invariance in growth processes," Adv. Phys. 46, 139 (1997).

${ }^{4}$ A. L. Barabasi and H. E. Stanley, Fractal Concepts in Surface Growth (Cambridge University Press, Cambridge, UK, 1995).

${ }^{5}$ G. Amir, I. Corwin, and J. Quastel, "Probability distribution of the free energy of the continuum directed random polymer in $1+1$ dimensions," Commun. Pure Appl. Math. 64, 466-537 (2011).

${ }^{6}$ P. L. Ferrari and H. Spohn, "Random growth model," in The Oxford Handbook of Random Matrix Theory, edited by G. Ackermann, J. Baik, and P. Di Francesco (Oxford University Press, 2011).

${ }^{7}$ T. Halpin-Healy and K. A. Takeuchi, "A KPZ Cocktail- Shaken, not stirred: Toasting 30 years of kinetically roughened surfaces,” J. Stat. Phys. 4, 794 (2015).

${ }^{8} \mathrm{~K}$. A. Takeuchi, "An appetizer to modern developments on the Kardar-ParisiZhang universality class," Physica A 504, 177 (2018), arXiv:1708.06060v3.

${ }^{9}$ H. S. Wio, "Variational formulation for the KPZ and related kinetic equations," Int. J. Bifurcation Chaos 19, 2813 (2009).

${ }^{10}$ H. S. Wio, R. R. Deza, C. Escudero, and J. A. Revelli, "Invited review: KPZ. Recent developments via a variational formulation,” Pap. Phys. 5, 050010 (2013).

${ }^{11}$ H. S. Wio, M. A. Rodriguez, R. Gallego, J. A. Revelli, A. Ales, and R. R. Deza, “ddimensional KPZ equation as a stochastic gradient flow in an evolving landscape: Interpretation, parameter dependence, and asymptotic form," Front. Phys. 4, 52 (2017).

${ }^{12}$ G. Gallavotti and E. G. D. Cohen, "Dynamical ensembles in nonequilibrium statistical mechanics," Phys. Rev. Lett. 74, 2694 (1995).

${ }^{13}$ G. Gallavotti and E. G. D. Cohen, "Dynamical ensembles in stationary states," J. Stat. Phys. 80, 931 (1995).

${ }^{14}$ J. Kurchan, "Fluctuation theorem for stochastic dynamics," J. Phys. A: Math. Gen. 31, 3719 (1998).

${ }^{15} \mathrm{G}$. E. Crooks, "Entropy production fluctuation theorem and the nonequilibrium work relation for free energy differences," Phys. Rev. E 60, 2721 (1999).

${ }^{16} \mathrm{U}$. Seifert, "Entropy production along a trajectory and an integral fluctuation theorem," Phys. Rev. Lett. 95, 040602 (2005).

${ }^{17}$ A. Imparato and L. Pelitti, "Fluctuation relations for a driven Brownian particle,” Phys. Rev. E 74, 026106 (2006).

${ }^{18}$ H. S. Wio, Path Integrals for Stochastic Processes: An Introduction (World Scientific, Singapore, 2013), ISBN:978-981-4374-78-1.

${ }^{19} \mathrm{~V}$. Y. Chernyak, M. Chertkov, and Ch. Jarzynski, "Path-integral analysis of fluctuation theorems for general Langevin processes," JSTAT 2006, P08001 (2006).

${ }^{20}$ U. Seifert, "Stochastic thermodynamics: Principles and perspectives," Eur. Phys. J. B 64, 423-431 (2008).

${ }^{21}$ U. Seifert, "Stochastic thermodynamics, fluctuations theorems and molecular machines," Rep. Prog. Phys. 75, 126001 (2012).

${ }^{22}$ C. Van den Broeck, "Stochastic thermodynamics: A brief introduction," in Proceedings of International School of Physics âĂIJEnrico Fermi"-Course CLXXXIV, Physics of Complex Colloids, edited by C. Bechinger et al. (IOS, Amsterdam, 2013).

${ }^{23}$ A. C. Barato, R. Chetrite, H. Hinrichsen, and D. Mukamel, "Entropy production and fluctuation relations for a KPZ interface," J. Stat. Mech. 2010, P10008 (2010).

${ }^{24}$ M. A. Rodriguez and H. S. Wio, "Stochastic entropies and fluctuation theorems for a discrete one-Dimensional Kardar-Parisi-Zhang system," Phys. Rev. E 100, 032111 (2019).

${ }^{25} \mathrm{O}$. Nieggemann and U. Seifert, "Field theoretic thermodynamic uncertainty relations - General formulation exemplified with the Kardar-Parisi-Zhang equation,” J. Stat. Phys. 178, 1142 (2020). arXiv:1908.05560 (2019).

${ }^{26} \mathrm{~B}$. Grossmann, H. Guo, and M. Grant, "Kinetic roughening of interfaces in driven systems,” Phys. Rev. A 43, 1727 (1991).

${ }^{27}$ T. Nattermann and L.-H. Tang, "Kinetic surface roughening. I. The KardarParisi-Zhang equation in the weak-coupling regime," Phys. Rev. A 45, 7156 (1992). 
${ }^{28} \mathrm{~B}$. M. Forrest and R. Toral, "Crossover and finite-size effects in the $(1+1)$-dimensional Kardar-Parisi-Zhang equation,” J. Stat. Phys. 70, 703 (1993).

${ }^{29}$ H. S. Wio, R. R. Deza, and J. A. Revelli, "Fluctuation theorems and largedeviation functions in systems not featuring a steady state," JSTAT 2020, P024009 (2020).

${ }^{30} \mathrm{H}$. Touchette, "A basic introduction to large deviations: Theory, applications, simulation,” Phys. Rep. 478, 1-69 (2009).

${ }^{31}$ S. N. Majumdar and A. Comtet, "Exact maximal height distribution of fluctuating interfaces,” Phys. Rev. Lett. 92, 225501 (2004).

${ }^{32}$ T. Gueudre, P. LeDoussal, A. Rosso, A. Henry, and P. Calabrese, "Short-time growth of a Kardar-Parisi-Zhang interface with flat initial conditions," Phys. Rev. E 86, 041151 (2012).

${ }^{33}$ M. Janas, A. Kamenev, and B. Meerson, "Dynamical phase transition in largedeviation statistics of the Kardar-Parisi-Zhang equation," Phys. Rev. E 94, 032133 (2016).
${ }^{34}$ P. Le Doussal, S. N. Majumdar, and G. Schehr, "Large deviations for the height in 1D Kardar-Parisi-Zhang growth at late times," Europhys. Lett. 113, 60004 (2016).

${ }^{35}$ B. Meerson and J. Schmidt, "Height distribution tails in the Kardar-ParisiZhang equation with Brownian initial conditions," J. Stat. Mech. 2017, 103207 (2017).

${ }^{36}$ Y. T. Fukai and K. A. Takeuchi, "Kardar-Parisi-Zhang interfaces with curved initial shapes and variational formula," Phys. Rev. Lett. 124, 060601 (2020).

${ }^{37}$ W. P. Gomez, A. L. A. Pena, and F. A. Oliveira, "From cellular automata to growth dynamics: The Kardar-Parisi-Zhang universality class," Phys. Rev. E 100, 020101(R) (2019).

${ }^{38}$ E. Marinari, A. Pagnani, and G. Parisi, "Critical exponents of the KPZ equation via multi-surface coding numerical simulations,” J. Phys. A 33, 8181 (2000).

${ }^{39} \mathrm{~S}$. G. Alves, T. J. Oliveira, and S. C. Ferreira, "Universality of fluctuations in the Kardar-Parisi-Zhang class in high dimensions and its upper critical dimension," Phys. Rev. E 90, 020103(R) (2014). 